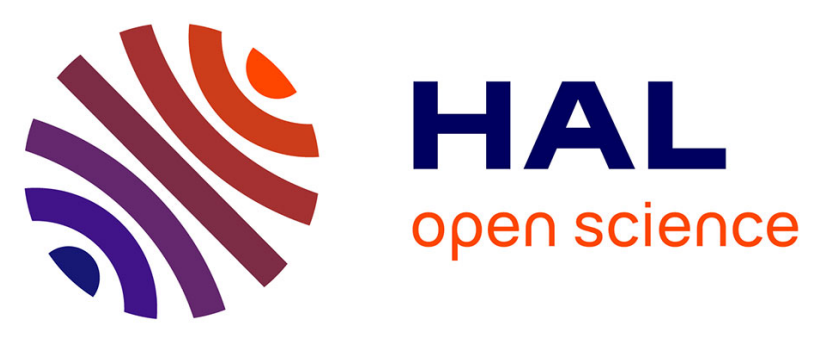

\title{
Beam shaping to enhance zero group velocity Lamb mode generation in a composite plate and nondestructive testing application
}

Frédéric Faëse, Samuel Raetz, Nikolay Chigarev, Charfeddine Mechri, James Blondeau, Benjamin Campagne, Vitali Goussev, Vincent Tournat

\section{To cite this version:}

Frédéric Faëse, Samuel Raetz, Nikolay Chigarev, Charfeddine Mechri, James Blondeau, et al.. Beam shaping to enhance zero group velocity Lamb mode generation in a composite plate and nondestructive testing application. NDT \& E International, 2017, 85, pp.13 - 19. 10.1016/j.ndteint.2016.09.003 . hal-01889431v2

\section{HAL Id: hal-01889431 \\ https://hal-univ-lemans.archives-ouvertes.fr/hal-01889431v2}

Submitted on 27 Apr 2019

HAL is a multi-disciplinary open access archive for the deposit and dissemination of scientific research documents, whether they are published or not. The documents may come from teaching and research institutions in France or abroad, or from public or private research centers.
L'archive ouverte pluridisciplinaire HAL, est destinée au dépôt et à la diffusion de documents scientifiques de niveau recherche, publiés ou non, émanant des établissements d'enseignement et de recherche français ou étrangers, des laboratoires publics ou privés. 
Zero group velocity (ZGV) Lamb modes have already shown their potential in nondestructive testing applications as they are sensitive to the sample structural characteristics. In this paper, we first consider an aluminum sample to validate a method based on the beam shaping of the generation laser. This method is proven to enhance ZGV Lamb modes in aluminum, and then advantageously applied to a composite material plate. Finally, based on the proposed method, scanning the sample over healthy and flawed zones demonstrates the ability to detect subsurface flaws.

\footnotetext{
${ }^{*}$ Corresponding author

** Principal corresponding author

Email addresses: samuel.raetz@univ-lemans.fr (Samuel Raetz), vincent.tournat@univ-lemans.fr (Vincent Tournat)

${ }^{1}$ Currently at: John A. Paulson School of Engineering and Applied Science, Harvard University, Cambridge, MA 02138, USA

Preprint submitted to NDTEEE International
} 


\section{Introduction}

Laser ultrasonics is a more and more widespread nondestructive testing method as it shows specific advantages compared to conventional ultrasonic methods based on transducers or EMATs. Particularly, it has a high spatial resolution, a large bandwidth, and it is non-contact [1]. Thanks to these features, laser ultrasonic techniques allow characterizing the mechanical properties and/or evaluating the structural health of materials, even where the tested samples present complex geometry and/or are subjected to extreme conditions such as high temperatures [2]. Up to now, applications of laser ultrasonic methods have already proved their potential in nondestructive testing of composite materials. They have been implemented successfully to detect delaminations with a propagative Lamb waves analysis [3] or by laser tapping [4]. They have also the ability to detect fiber breakage or matrix cracking via the scanning laser source technique [5] or even porosity thanks to an ultrasonic spectroscopy method [6].

Guided waves have been used in composite samples testing and evaluation because of their ability to detect a defect at a long propagation distance from the acoustic source position. Yet, the defect position is hard to precisely estimate at a single interface at any position through the laminate [3]. As well, a lateral position estimation needs methods with a transducer raster scan like the SAFT method [7] or transducer arrays like the topological imaging technique [8]. For ten years, some specific non-propagative Lamb modes called zero group velocity (ZGV) Lamb modes have been studied and already applied to defect detection. Considering a dispersion curve representing the angular frequency $\omega$ as a function of the wave number $k$, these specific modes 
are located in the points of a non-zero wave number $k$ where the slope of this curve is horizontal, i.e. $\mathrm{d} \omega / \mathrm{d} k=0$. Characterized by a high quality factor, these modes are used for instance to measure thickness variations due to corrosion, to detect disbonding or to determine elastic constants $[9,10]$. A method based on the ZGV Lamb modes offers the advantages of being local and having a spatial resolution of the order of the plate thickness [10]. One objective of this article is to report on the effect of a flaw in a composite plate on ZGV Lamb modes.

The challenges to address when generating ZGV Lamb modes in composite plates are numerous. Firstly, the composite plates usually have a low damage threshold. For instance, the sample used in this paper showed fiber whitening at about $5 \mathrm{MW} . \mathrm{cm}^{-2}$ with a 1064 nm-wavelength laser, whereas the threshold for aluminum in the same experimental conditions is about 50 MW.cm ${ }^{-2}$ [11]. Secondly, as composite surfaces are matt and diffusive for light, ultrasonic waves are hardly detectable using non-contact optical techniques. Thirdly, quality factors of ZGV Lamb modes are strongly reduced because of the resin viscoelasticity leading to a strong sound attenuation in composite plates. Note that ZGV Lamb modes could even not exist, regarding the anisotropic mechanical properties of the material; nevertheless, this is out of the focus of this paper.

In this work, elastic waves are generated in an aluminum plate or a composite plate by a pulsed laser and detected using an interferometer. By varying the focusing of the pump laser beam, it is possible to favor the generation of either the propagating modes or the ZGV modes in the plate. Especially, considering a circular laser spot as the thermoelastic source, ZGV 
Lamb modes are efficiently excited when the spot radius is about the plate thickness [12]. It is first shown that ZGV Lamb modes can be enhanced or reduced in an aluminum plate thanks to a specific beam shaping of the generation laser [13-17]. Then, the same method is advantageously applied to the healthy zone of a composite plate. Finally, a damaged region of the same composite plate is considered and the influences of the flaw on the ZGV Lamb modes are analyzed. Before going into details, the mechanical properties of the tested sample and the experimental setups are introduced.

\section{Mechanical properties of the tested sample and experimental setups}

This section is devoted to the analysis of the influence of flaws on ZGV Lamb modes in a plate made of composite material. As the setup is based on a beam-shaping mask to selectively generate a specific ZGV mode, it is important to know the mechanical properties of the tested material in order to design the beam-shaping mask. We will show that the optimal geometrical properties of the beam-shaping mask for a particular ZGV mode generation depend on the ZGV wavelength in the sample. In the framework of industrial nondestructive testing (NDT), mechanical properties of the composite materials are already well characterized. Hence, the wavelengths of the ZGV modes that can be generated in the sample are known. Here the mechanical properties of the composite sample were first characterized in order to determine an interesting ZGV mode and then to make the appropriate beamshaping mask.

In order to predict the dispersion curve of the composite plate, both the density and the elastic constants have to be determined. First, the volu- 
metric mass density of the composite sample has been estimated thanks to Archimedes principle to be $\rho_{\text {comp }} \approx 1540 \mathrm{~kg} \cdot \mathrm{m}^{-3}$. Second, the elastic constants have been determined thanks to a method explained in Refs. [18] and [19]. Based on appropriate A-scans, plane wavefronts are synthesized by summing the signals with suitable delays. By changing the synthetized propagation angle, quasi-longitudinal and quasi-transversal time of flights are semi-automatically recorded. Finally, the theoretical slowness curve that fits the experimental one is determined, thanks to a minimization method. This leads to the following estimation of the elastic constants: $C_{11}=13.4 \mathrm{GPa}$, $C_{12}=3.00 \mathrm{GPa}, C_{22}=21.36 \mathrm{GPa}$, and $C_{55}=3.64 \mathrm{GPa}$. Note that these constants have been obtained by assuming that the composite plate is transversely isotropic relative to an axis normal to the surface. The composite plate is a carbon fiber-epoxy composite, composed of 10 plies of $300 \mu$ m-thick each, oriented as [45/0/0/45/0/45/0/45/0/45]. Thanks to the SAFE method, the composite plate structure was confirmed to be transversely isotropic. Finally, from the measured plate thickness, $d_{\text {comp }} \approx 3.2 \mathrm{~mm}$, the dispersion curves have been calculated and are displayed in Fig. 1. As one could find in literature [20, 21], ZGV Lamb mode can occur in anisotropic material and the wavelength of the first ZGV Lamb mode in our composite plate (cf. vertical arrow in Fig. 1 is here estimated with a precision that is sufficient for the method we propose: $\lambda_{Z G V}^{\operatorname{comp}} \approx 15.3 \mathrm{~mm}$.

Due to the low damage threshold of composite materials, the choice has been done to experimentally use an extended line source instead of a circular spot, in order to lower the source power density on the sample while keeping sufficiently large displacement amplitude. Theoretically, using an infinitely 


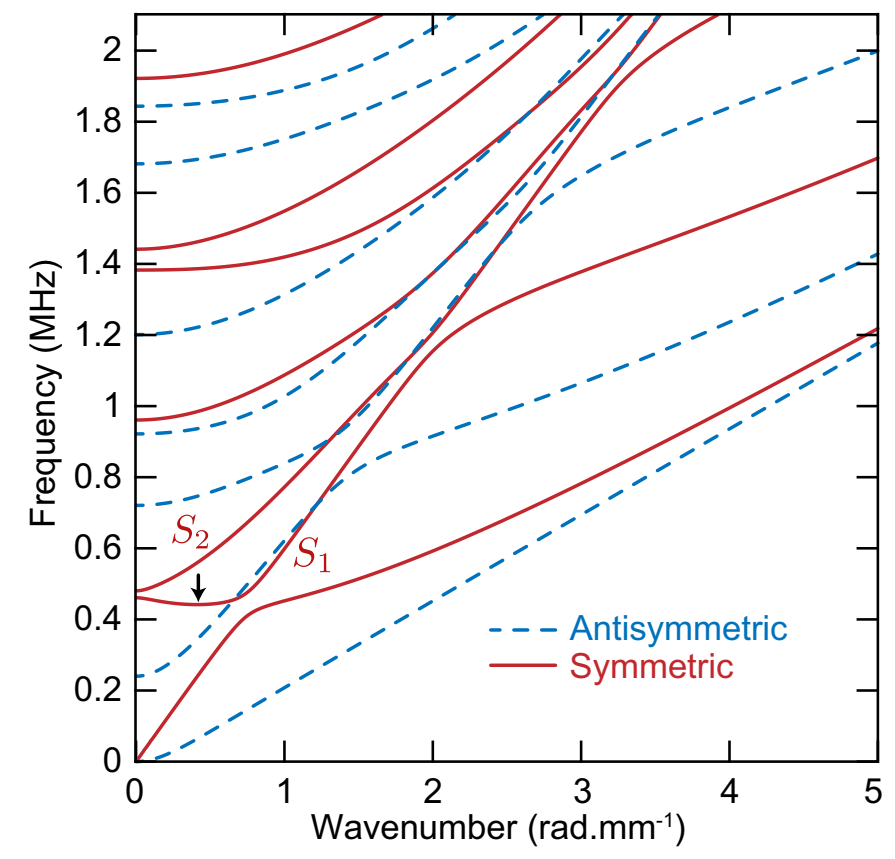

Figure 1: (color online) Dispersion curves of the symmetric (solid line) and antisymmetric (dashed line) Lamb modes calculated in a 3.2-mm-thick composite plate. The first ZGV mode is shown by the vertical arrow and has a wavelength of $\lambda_{Z G V}^{c o m p} \approx 15.3 \mathrm{~mm}$.

long and thin thermoelastic line source, the amplitude of the surface displacement due to a ZGV Lamb mode as a function of the distance from the line source is a pure cosine function, since it results from the interference of two counter-propagative Lamb modes having the same wave number, i.e. of the form $e^{j k_{Z G V} x}$ and $e^{-j k_{Z G V} x}$. In the case of a finite line source, the amplitude varies as an intermediate function between the pure cosine function and a Bessel function [22], the latter standing for the theoretical spatial distribution of ZGV Lamb modes in the case of a point surface source [12]. In order to selectively generate the first ZGV Lamb mode and to increase the total incident laser power while keeping the power density constant, we propose here 
to use multiple finite line sources using an appropriate beam-shaping mask. As illustrated in Fig. 2, using the beam-shaping mask allows the generation laser beam to be shaped with periodic slits whose spacing matches the ZGV wavelength, thereby producing a constructive interference of the ZGV Lamb modes generated by each line source. Note that the laser transient grating technique[13-15] is another efficient technique to selectively generate elastic guided waves with a given wavelength. The use of an optical mask instead of the laser transient grating technique has been mainly chosen for the reason of experimental simplicity. Achieving a transient laser grating with such large period would have indeed required the laser beams to be crossed with a really small angle (about $2.10^{-3} \mathrm{deg}$ ), which was technically difficult to reach with a good precision.

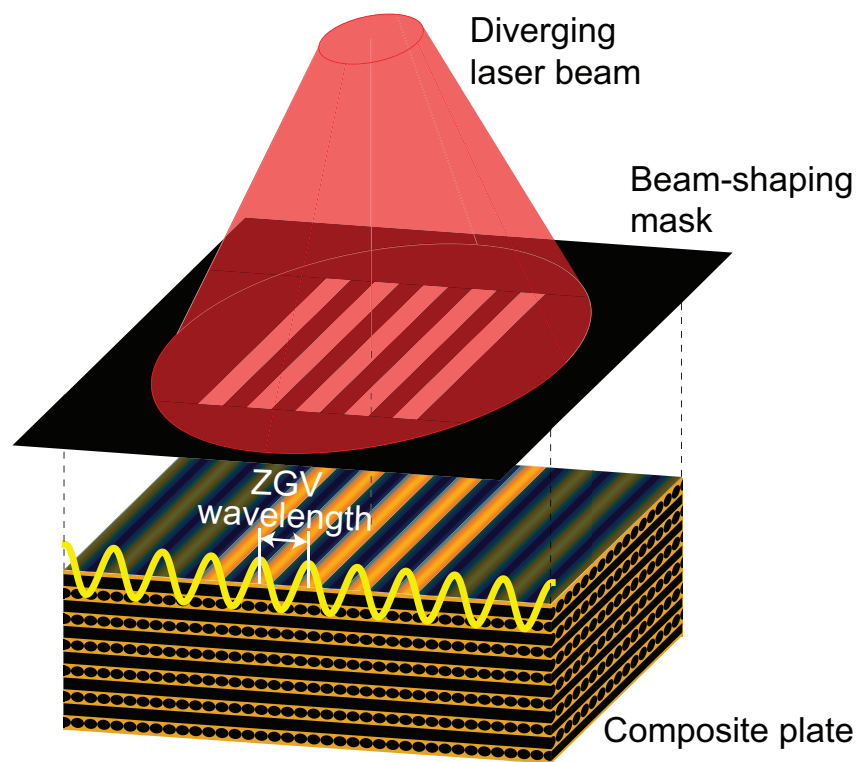

Figure 2: (color online) Illustration of the ZGV Lamb mode generation using a beamshaping mask. The generation laser beam is shaped with periodic slits whose spacing matches the ZGV wavelength. 
In order to analyze the ZGV Lamb mode generation using a shaped laser beam, the experimental setup shown in Fig. 3 has been implemented. The generation laser is an Nd:YAG laser (Spitlight Compact 400, InnoLas Laser GmbH, Germany) emitting $10 \mathrm{~ns}$ pulses at $1064 \mathrm{~nm}$. The pulse energy is limited to a few tens of $\mathrm{mJ}$ in order to remain below the damage threshold and to avoid fiber whitening. This limitation of the laser energy is performed using two polarizing beam splitters (PBS) and a half-wave plate $(\lambda / 2)$ as shown in Fig. 3. Thanks to a negative lens, the whole beam shaping mask is illuminated by the generation laser. This mask consists of a series of transparent and opaque patterns (Fig. 2) printed on transparency films by a laser printer. Two identical masks are placed one after the other in the close vicinity of the plate in order to obtain a sufficient contrast. The normal surface displacement due to ultrasonic waves is detected on the other side of the sample by a two-wave mixing (TWM) interferometer (LU-TWM-ASGA, Tecnar Canada) whose bandwidth ranges from about $1 \mathrm{MHz}$ to $40 \mathrm{MHz}$ [23]. Note that the bandpass spectrum of the interferometer filter is smooth and still allows to detect displacement with frequency component down to about $300 \mathrm{kHz}$. The TWM is using a CW Nd:YAG laser, the beam of which is guided through an optical fiber to the TWM head including a neutral density filter and the focusing/collecting lens. The TWM head is mounted on a motorized linear stage so that the detection point is scannable over the sample. A second motorized linear stage can also be used to scan the sample in front of fixed generation pattern and detection point. Since generation and detection of elastic waves are on opposite sides, this setup is referred to as the transmission setup in the following. 


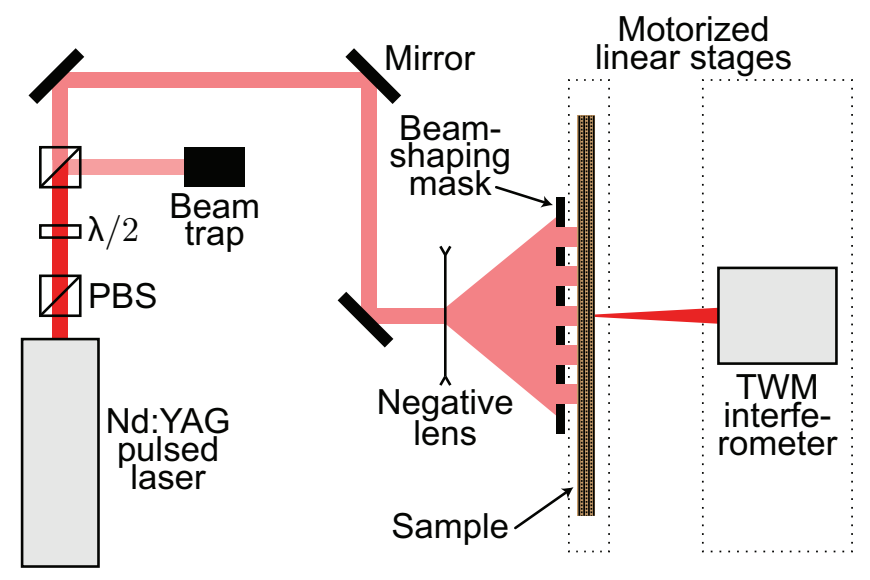

Figure 3: (color online) Schematics of the experimental transmission setup.

A second setup has been used in this study, with generation and detection on the same side, which is referred to as the reflection setup in the following. It is identical to the transmission setup, except that both generation laser beam and TWM laser beam illuminate the same side of the sample, and that the generation laser beam is frequency doubled in order not to dazzle the TWM interferometer photodiode that is sensitive to $1064 \mathrm{~nm}$ radiation. This setup can particularly be useful for a robot inspection and can give additional information for a flaw characterization as it will be discussed in the following: where the transmission setup detects a flaw without information on its in-depth position, we will see that the reflection setup is able to give information on the flaw position with respect to the depth.

Before applying the proposed method to the detection of a flaw in a composite plate, it is first proposed to focus on the interest of using a beamshaping mask to enhance the ZGV Lamb modes in two samples: first, an aluminum plate for trivial evidence, and then a composite plate. 


\section{ZGV Lamb modes enhancement in aluminum and composite plates}

In order to validate preliminary results obtained with the transmission setup shown in Fig. 3, Fig. 4 presents the experimental results obtained in an $4.1 \mathrm{~mm}$-thick aluminum plate using a single thermoelastic line source of dimensions $4.1 \times 20 \mathrm{~mm}^{2}$. Figs. 4(a)-(b) are slightly saturated in order to improve readability.

The time domain B-scan [Fig. 4(a)] represents the normal surface displacement amplitude as a function of time and the TWM head position. The signals have been registered over $200 \mu$ s with the TWM head position ranging from $-50 \mathrm{~mm}$ to $+50 \mathrm{~mm}$ with a $0.5 \mathrm{~mm}$ step. Two different kinds of modes are visible: (i) the propagating modes starting at the origin in time and space, and (ii) the ZGV Lamb modes that are visible in time after the propagating modes. The ZGV modes are evidenced by a succession of maxima and minima in time and space, typical of the single frequency and the interferential nature of the ZGV modes.

The frequency domain B-scan [Fig. 4(b)] represents the spectral amplitude module of each A-scan constituting the time domain B-scan as a function of the TWM head position. Each spectrum constituting the frequency domain B-scan has been calculated over the whole corresponding A-scan. At the first, i.e. lowest, expected ZGV Lamb mode frequency, $f_{Z G V}^{A l} \approx 694 \mathrm{kHz}$, there is a maxima and minima succession in space that is typical of ZGV Lamb modes obtained with a thermoelastic line source. When zooming at the ZGV frequency [Fig. 4(c)], the first ZGV peak amplitude as a function of the TWM head position (solid) shows the expected theoretical shape 


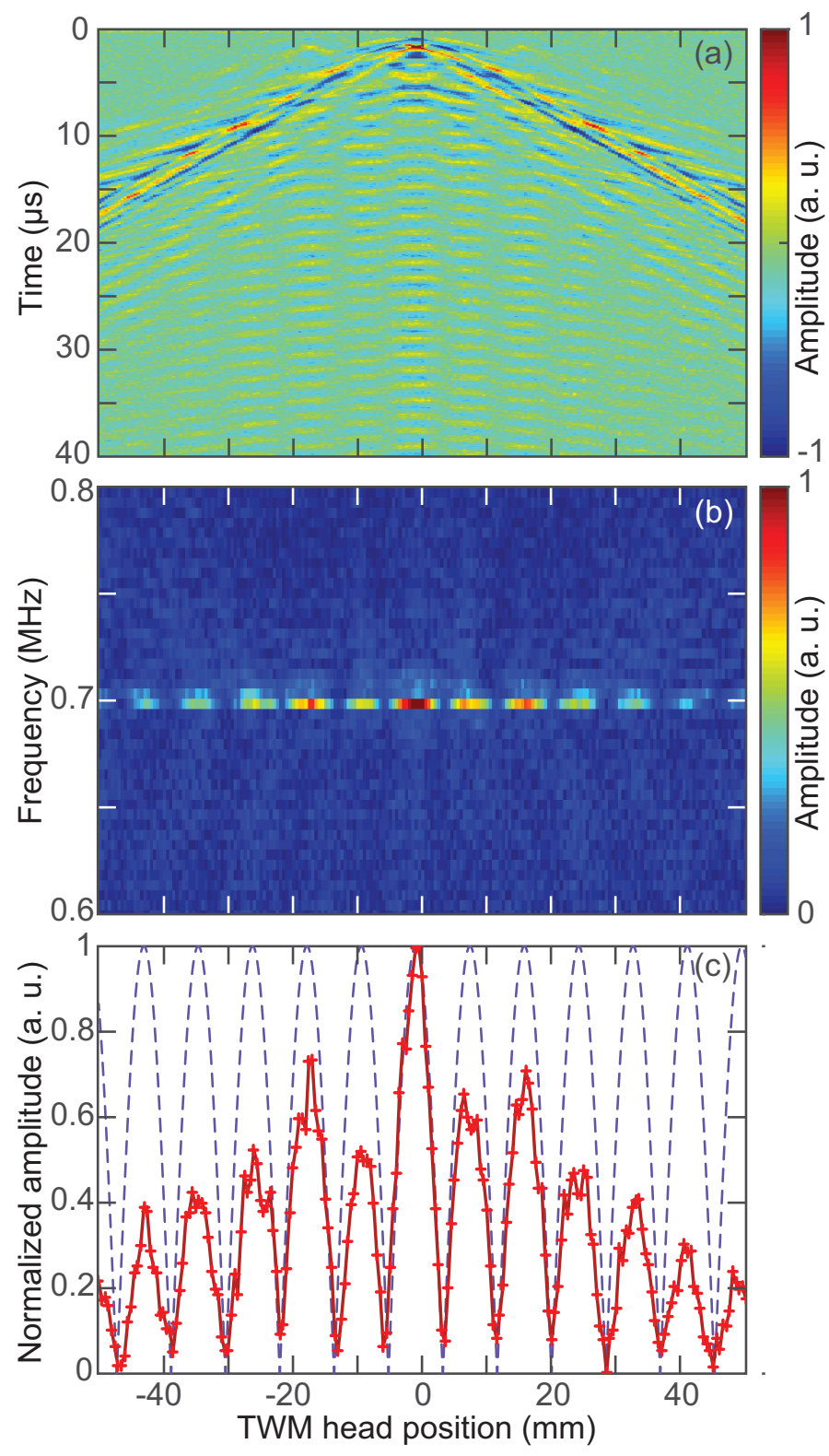

Figure 4: (color online) The thermoelastic source is a line $4.1 \times 20 \mathrm{~mm}^{2}$ : (a) time domain and (b) frequency domain B-scans as a function of the TWM head position. (c) First ZGV peak amplitude vs. TWM head position: experimental (solid) and theoretical (dashed) curves. 
(dashed), especially regarding the minima. As expected theoretically, the amplitude of the experimental curve decays with the distance from the line source position because of the line source finite dimensions.

It is now proposed to compare this result obtained with a single line source to the results obtained with a shaped source composed of lines spaced by either $\lambda_{Z G V}^{A l}$ or $\lambda_{Z G V}^{A l} / 2$. Figure 5 presents the amplitude of the first ZGV peak as a function of the TWM head position when the thermoelastic source is made of: a single line (solid), multiple lines spaced by $\lambda_{Z G V}^{A l}$ (dashed), and multiple lines spaced by $\lambda_{Z G V}^{A l} / 2$ (dash-dotted). The Frobenius norm (Euclidian norm) of each normal displacement field is used to normalize each corresponding curve in Fig. 5, in order for the changes in the absorbed laser power between the different cases to be compensated.

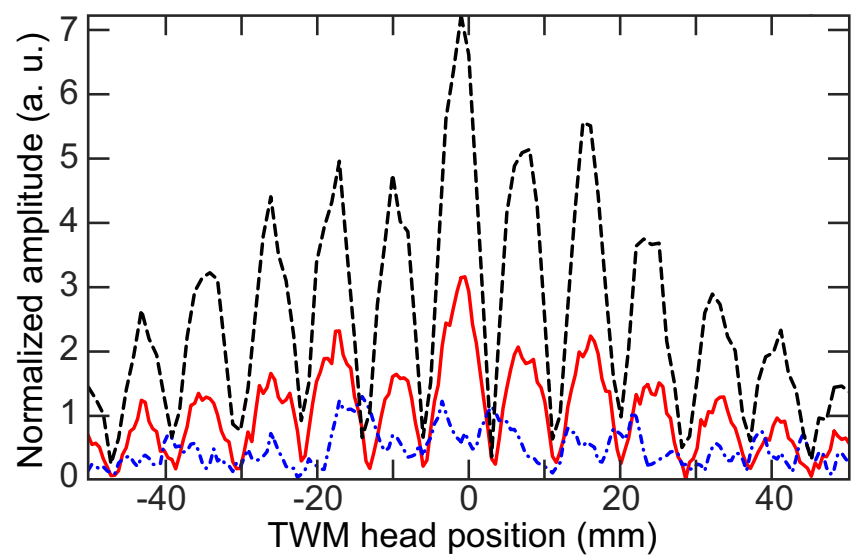

Figure 5: (color online) Normalized first ZGV peak amplitude vs. TWM head position when the thermoelastic source is a single line (solid), multiple lines spaced by $\lambda_{Z G V}^{A l}$ (dashed) and multiple lines spaced by $\lambda_{Z G V}^{A l} / 2$ (dash-dotted).

When the lines are spaced by $\lambda_{Z G V}^{A l}$, the ZGV Lamb modes are enhanced: they interfere constructively. On the contrary, when the lines are spaced 
by $\lambda_{Z G V}^{A l} / 2$, the ZGV Lamb mode amplitudes are reduced: they interfere destructively. As expected, a mask consisting of slits spaced by the ZGV wavelength enhances the ZGV Lamb modes generation in aluminum compared to a mask consisting of a single slit. The interest of this beam-shaping method being demonstrated in a metal plate, it is now examined in the more challenging case of a composite plate.

The beam-shaping mask used with the composite plate consists of three slits spaced by $\lambda_{Z G V}^{c o m p}$. Figures 6(a)-(c) show the experimental results. Figure 6(a) stands for the time domain B-scan, while Fig. 6(b) shows the frequency domain B-scan. Figures 6(a)-(b) are slightly saturated in order to improve readability. The amplitude of the first ZGV peak with respect to the TWM head position obtained for the three-line source (thick line) is compared to the same amplitude obtained with a single line source (thin line) in Fig. 6(c). The ZGV peak amplitudes in Fig. 6(c) are normalized following the same method as the one described for aluminum. The comparison between the thin and thick lines in Fig. 5(c) clearly demonstrates that the ZGV peak is almost not observable when one slit is used. This emphasizes that it is preferable to use three slits rather than one for the investigation of a composite plate in order to increase the generation efficiency of the ZGV mode and to get a sufficiently large signal to noise ratio for this particular mode. Note that it is not the case for the Aluminum, where the ZGV mode is well detectable when using one slit (cf. Fig. 4).

The time domain B-scan [Fig. 6(a)] highlights the propagating modes and we slightly see the ZGV Lamb modes. The frequency domain B-scan [Fig. 6(b)] points out the ZGV Lamb modes at the frequency $f_{Z G V}^{\text {comp }} \approx 0.480 \mathrm{MHz}$ 
which is confirmed by Fig. 6(c). Indeed, as ZGV peaks correspond to energy peaks, they are expected to be spaced by half the ZGV wavelength, that is to say that ZGV peaks are expected to be located in the middle of each illuminated slits as well as centrally located between two adjacent slits. As Fig. 6(c) shows these peaks on the slits location (under black arrows) and centrally located between two adjacent slits (under gray arrows), this result tends to prove the enhancement of the ZGV mode in the composite plate thanks to the proposed beam-shaping mask.

In order to obtain Fig. 6(c), the ZGV peak amplitude was measured as the maximum spectral amplitude module between $0.475 \mathrm{MHz}$ and $0.485 \mathrm{MHz}$. This frequency range can be related to a possible sample thickness variation since the product of the ZGV mode frequency $f_{Z G V}$ by the sample thickness is constant. Hence, the average ZGV frequency $\left(f_{Z G V}\right)_{0}$ and the ZGV frequency variation $\Delta f_{Z G V}$ on the one hand, and the average sample thickness $d_{0}$ and the sample thickness variation $\Delta d$ on the other hand, are related to each other:

$$
\frac{\Delta d}{d_{0}}=-\frac{\Delta f_{Z G V}}{\left(f_{Z G V}\right)_{0}} .
$$

The range [0.475-0.485] $\mathrm{MHz}$ leads to a thickness variation $\Delta d$ of about $\pm 33 \mu \mathrm{m}$. This sample thickness variation is very small and it is consistent with the precision of the sample fabrication process.

As the beam-shaping method is validated to enhance ZGV Lamb modes in the composite plate, a suitable mask with slits spaced by $\lambda_{Z G V}$ is now used for an NDT application. 


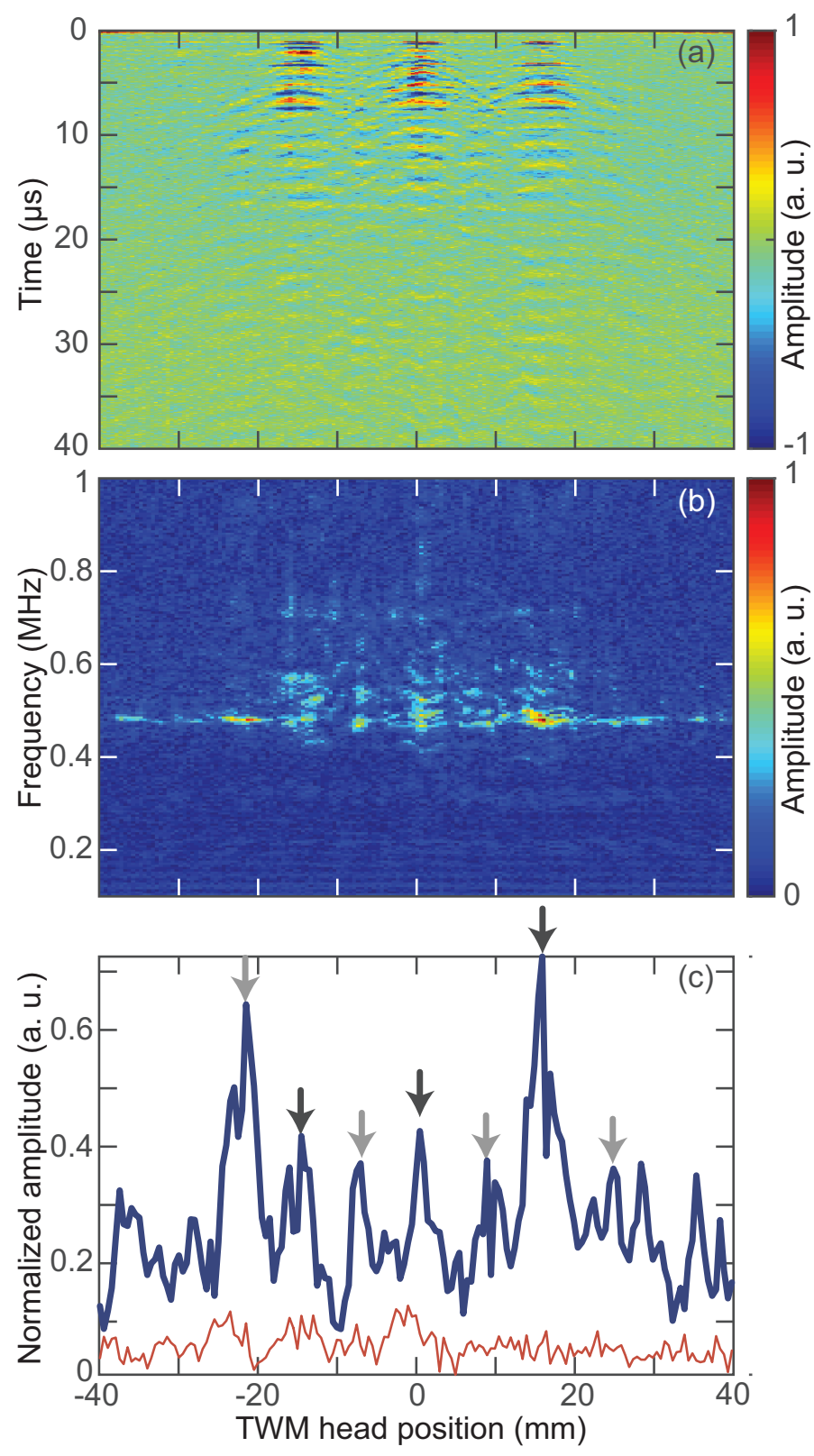

Figure 6: (color online) Results obtained in the composite plate with a thermoelastic source made of three lines: (a) time domain and (b) frequency domain B-scans vs. TWM head position. (c) Normalized amplitude of the first ZGV peak vs. TWM head position (thick line) compared with the result obtained with a single line source (thin line). 


\section{NDT application in a composite plate}

The reflection setup described in Sec. 2 is now used to scan the composite plate in front of both spatially fixed Nd:YAG frequency doubled laser beam and TWM interferometer beam. The former is shaped by a mask having three slits spaced by $\lambda_{Z G V}^{c o m p}$ and the latter being focused to a point in the middle of the beam shaping mask (middle line). As quickly explained in Sec. 2, the choice of using a reflection setup instead of the so-far used transmission setup is twofold: (i) this is closer to industrial applicability, and (ii) the flaw in the tested sample is such that, at the flaw location, there is no direct transmission of the elastic waves through the flaw, making the lateral detection of the flaw easy but not the in-depth location. We will see that using a reflection setup can lead to this in-depth characterization of the flaw.

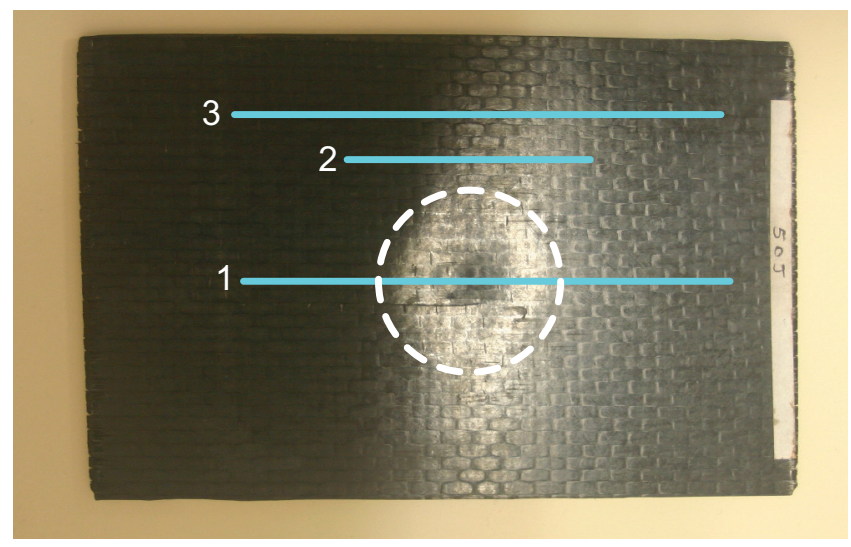

Figure 7: (color online) Composite sample and representation of the scan lines (solid). Dashed white line circle: maximum size of the flaw observed optically.

The composite sample presented in Fig. 7 has been impacted by a $50 \mathrm{~J}$ centered shock. The $50 \mathrm{~J}$ shock was obtained thanks to the drop of a hemispherical mass with a diameter of $25 \mathrm{~mm}$. The sample was clamped on a 

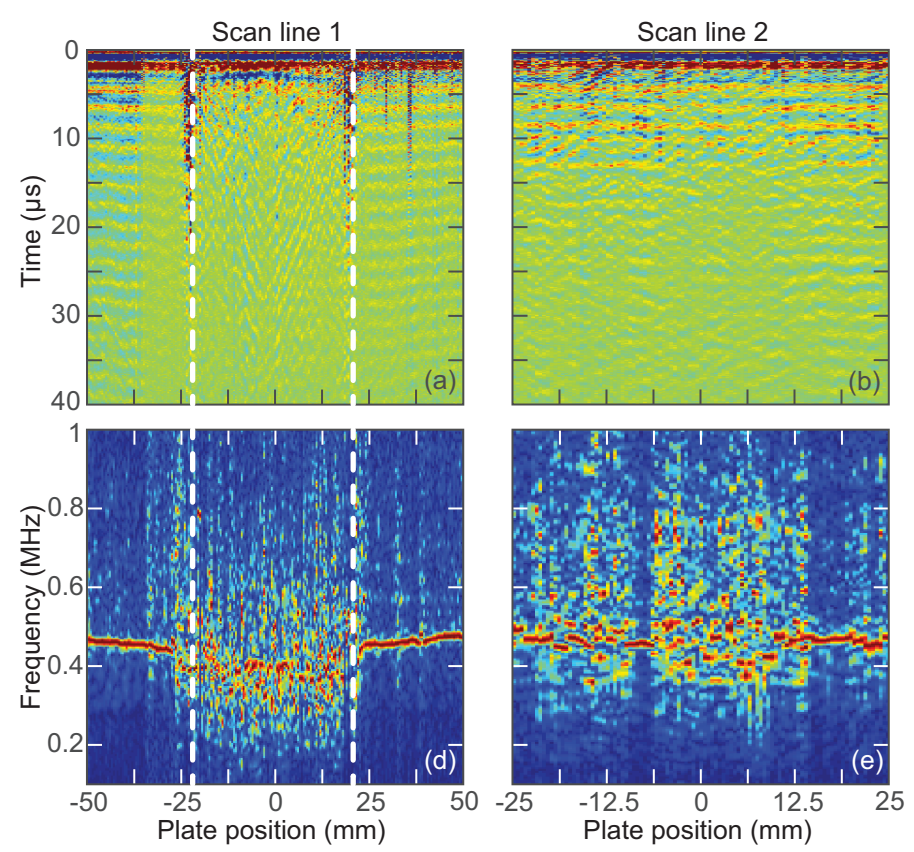

bearing having a $40 \mathrm{~mm}$-diameter hole, centered with respect to the drop mass axis. The first scan line (line 1) crosses the impact whereas the second scan line (line 2) is near the impact location and the third scan line (line 3 ) is far from the impact location. Within all these scans, the line sources composing the shaped thermoelastic source were perpendicular to the scan direction. Regarding line 3, note that the shaped thermoelastic source is at least $5 \mathrm{~mm}$ away from both the plate edge and the impact location, whatever the plate position during the scan is.

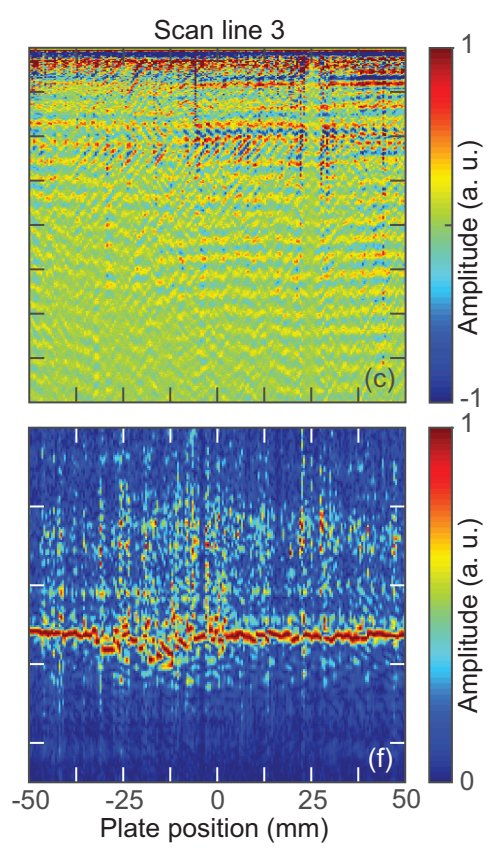

Figure 8: (color online) Time domain B-scans of the composite plate when the scan line is: line 1 across the impact location (a), line 2 near the impact location (b), and line 3 far from the impact location (c). The related frequency domain B-scans obtained with a Hann time window (respectively d, e and f). Dashed white lines: maximum dimension of the flaw observed optically.

The time domain B-scan signals have been registered over $200 \mu$ s with 
the plate position ranging from $-50 \mathrm{~mm}$ to $+50 \mathrm{~mm}$ [Fig. 8(a) and Fig. 8(c)] or from $-25 \mathrm{~mm}$ to $+25 \mathrm{~mm}$ [Fig. 8(b)] with a $0.5 \mathrm{~mm}$ step. Each A-scan for a given plate position is numerically post-processed in order to have a zero mean value over the useful signal duration, i.e. from $10 \mu$ s to $100 \mu$ s. The frequency domain B-scans have then been calculated over the whole time domain signals filtered by a Hann time window ranging from $0 \mu$ s to $100 \mu \mathrm{s}$ in order to emphasize the ZGV Lamb modes. The spectrum amplitude for a given plate position is normalized to its maximum.

In Fig. 8(a), the time domain B-scan across the impact location highlights the impact edges marked by the dashed white lines (cf. also Fig. 7). Moreover, regular successions of maxima and minima in time, representative of ZGV oscillations, can be observed outside of the impact location (in a healthy zone) whereas the signal appears disturbed inside the impact location. The time domain B-scans near the impact location and far from the impact location [Fig. 8(b) and Fig. 8(c), respectively] show minor changes with the plate position and no flaw is clearly evidenced.

The frequency domain B-scan across the impact location [Fig. 8(d)] also clearly highlights the impact as only the ZGV frequency is visible outside the impact location whereas on the impact location the dominant frequency components are spread out over the range $\sim 200-800 \mathrm{kHz}$ and show quick variations in space. Let us also notice that the ZGV frequency decreases almost linearly when the signal measurement gets closer to the impact. As the product $f_{Z G V}$ by the thickness is constant, this shows that either the plate thickness increases with the impact vicinity (the $50 \mathrm{~J}$ impact has lead to a bulge at the vicinity of the impact) or the elastic moduli diminish. 
The frequency domain B-scan near the impact location [Fig. 8(e)] also shows changing multiple frequency components between $-5 \mathrm{~mm}$ and $+15 \mathrm{~mm}$ that could be due to the impact vicinity. Moreover, farther from the impact location, i.e. between $-25 \mathrm{~mm}$ and $-10 \mathrm{~mm}$, multiple frequency components also appear whereas this zone should be healthy. The frequency domain Bscan far from the impact location [Fig. 8(f)] shows that the main frequency component is the ZGV frequency below $-30 \mathrm{~mm}$ and above $0 \mathrm{~mm}$; these zones can thus be considered as quasi-healthy zones. On the contrary, between $-30 \mathrm{~mm}$ and $0 \mathrm{~mm}$, a hidden flaw is detected. This confirms the previous result [Fig. 8(e)] highlighting a flaw on a zone that should be healthy.

These results have been compared to the composite plate inspection obtained with a system called LUCIE, the technical specifications of which are gathered in Ref. [24]. Note that the minimum laser fluence delivered by the pump laser $\left(\mathrm{CO}_{2}, 270 \mathrm{~mJ}, 100 \mathrm{~ns}\right)$ in the LUCIE system is $1.5 \mathrm{MW} . \mathrm{cm}^{-2}$, which is below the damage threshold of the composite. Signals are gathered at a fast rate by the LUCIE system thanks to its scan head which includes both the pump laser and the probe laser used for the interferometric measurement of the sample surface velocity. Figure 9 represents the C-scan inspection of the composite plate consisting in measuring the ratio of two ultrasonic echo amplitudes as a function of the position on the plate: the ratio of the amplitudes of the second ultrasonic echo detected (acoustic waves reflected by the rear surface of the plate or by a defect) to the first ultrasonic echo detected (corresponding to the surface expansion due to laser absorption). Note that the ratio can be done after filtering the collected raw data with a selective band-pass filter. LUCIE's data post-processing may also give 
an image (not shown in this paper) of the time of flight difference between the first and the second detected acoustic echo, although this image is not precise for in-depth localization of defects close to the surface. The presented scan (Fig. 9) shows two major results. First, the $50 \mathrm{~J}$ impact is very well identified by the centered zone that shows the lowest value. Second, on the healthy zone scanned previously (cf. line 3 in Fig. 7), the ratio has either values in [4.55-9.75] \% that should be indicative of a real healthy zone or weak values lower than $3.25 \%$ that are indicative of a flaw. This last result confirms the presence of the hidden flaw detected previously along the scan line 3 by the method proposed by the authors.

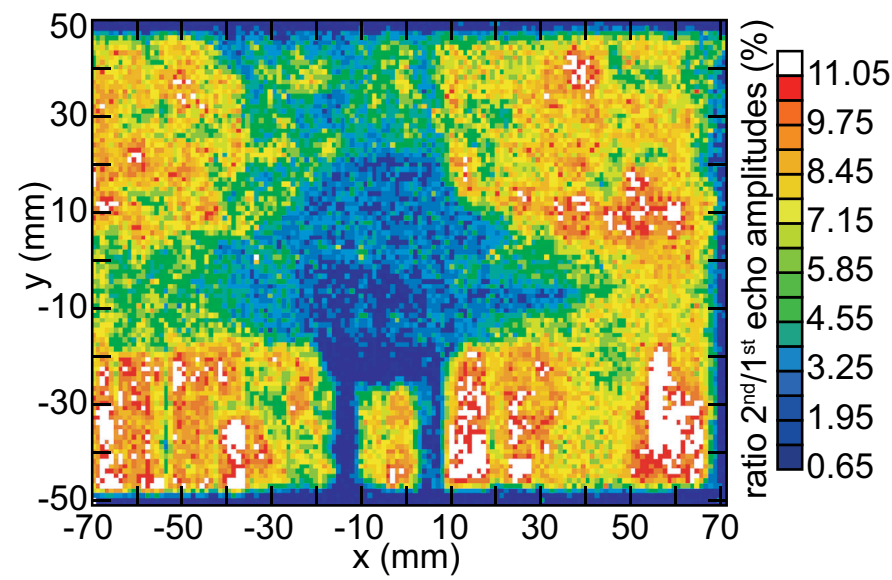

Figure 9: (color online) C-scan inspection of the composite plate obtained with the LUCIE system: ratio of the second ultrasonic echo amplitude to the first ultrasonic echo amplitude

Using the experimental transmission setup, a supplementary scan has been done along the scan line 1 , the results of which are not presented in this paper. The observation is that, at the flaw location, no elastic wave is directly transmitted. This observation probably means that the $50 \mathrm{~J}$ impact 
produced a delamination inside the composite plate.

Let us consider that the impact produced a delamination inside the composite plate, hence splitting the plate into a minimum of two thinner plates one above the other. If we assume that laser generated ultrasonic waves interact only with the top plate, several mechanisms can explain why the frequency domain B-scans show multiple frequency components on the delamination location. A given frequency component different from the ZGV mode frequency of the plate can be due to a membrane resonance effect [25] or to a local defect resonance [26]. It could even be due to another ZGV mode frequency associated to a smaller thickness than the one of the plate. A flaw resonance frequency $f_{r}$ can be estimated by assuming a circular clamped membrane:

$$
f_{r}=\frac{1.88 h}{d^{2}} \sqrt{\frac{E}{\rho\left(1-\nu^{2}\right)}},
$$

where $h$ and $d$ are the flaw depth and diameter respectively, and $E, \rho$ and $\nu$ are the sample Young's modulus, density and Poisson ratio respectively.

Assuming that the characteristic frequency is due to a circular flaw resonance and using the following material parameters: $h=300 \mu \mathrm{m}$ (a ply thickness), $E=15 \mathrm{GPa}, \rho=1540 \mathrm{~kg} / \mathrm{m}^{3}$, and $\nu=0.2$, the flaw dimension could be estimated as $d=2.1 \mathrm{~mm}$, the flaw resonance being in that case $f_{r} \simeq 0.41 \mathrm{MHz}$. This estimates is in good agreement with the frequency values observed in the frequency domain B-scans in Fig. 8. More information on the material parameters and possible flaw geometry is necessary. We believe however that in the near future the development of a realistic model could lead to quantitative estimates of defect parameters relevant for the structural health assessment of composite structures. 


\section{Conclusion}

We have presented a method enabling ZGV Lamb modes enhancement thanks to the beam shaping of the generation laser, making use of a mask with slits spaced by the ZGV wavelength. First, ZGV Lamb modes have been analyzed in an aluminum plate to validate the possibility to enhance or reduce their amplitude depending on the thermoelastic line sources spacing. It has been shown that ZGV Lamb modes interfere constructively when the line sources spacing equals the ZGV wavelength whereas they interfere destructively when the spacing is halved.

After the thorough characterization of a composite plate that resulted in the ZGV wavelength determination, the beam-shaping method has been applied to the composite sample. Experimental results demonstrate a ZGV Lamb mode enhancement, hence validating the beam-shaping method also in the case of a composite sample. Finally, by scanning over the sample, the measured signal frequency content near the ZGV frequency has proven its ability to distinguish between healthy zones and an impacted zone of a composite plate, and also to detect flaw zones that are not visually detectable. The next step could possibly be to extract quantitative information from the dominant frequencies of the B-scans compared to those predicted by the mechanical models of the defects.

\section{Acknowledgments}

This presentation is part of the LUCITA project managed by IRT Jules Verne (French Institute in Research and Technology in Advanced Manu-

facturing Technologies for Composite, Metallic and Hybrid Structures). The 
authors wish to associate the industrial and academic partners of this project:

Airbus Group Innovations and STELIA Aerospace; EMN-Subatech and LAUM respectively.

\section{References}

[1] J. Cooper, R. A. Crosbie, R. J. Dewhurst, A. D. W. McKie, and S. B. Palmer, Surface acoustic wave interactions with cracks and slots: A noncontacting study using lasers, IEEE Trans. Ultrason. Ferroelectr. Freq. Control. 33, 462-470 (1986).

[2] D. W. Schindel, D. A. Hutchins, S. T. Smith, and B. Farahbakhsh, Hightemperature pulsed photoacoustic studies of surface waves on solids, J. Acoust. Soc. Am. 95, 2517-2524 (1994).

[3] N. Guo and P. Cawley, The interaction of Lamb waves with delaminations in composite laminates, J. Acoust. Soc. Am. 94, 2240-2246 (1993).

[4] A. Blouin, C. Néron, B. Campagne, and J.-P. Monchalin, Applications of laser-ultrasonics and laser-tapping to aerospace composite structures., in Conference proceedings of 17th WCNDT. Shanghai., page 7 (2008).

[5] P. A. Fomitchov, A. K. Kromin, S. Krishnaswamy, and J. D. Achenbach, Imaging of damage in sandwich composite structures using a scanning laser source technique, Compos. Part B-Eng. 35, 557-562 (2004), marine Composites.

[6] A. A. Karabutov and N. B. Podymova, Quantitative analysis of the influence of voids and delaminations on acoustic attenuation in CFRP 
composites by the laser-ultrasonic spectroscopy method, Compos. Part B-Eng. 56, 238-244 (2014).

[7] M. Spies and W. Jager, Synthetic aperture focusing for defect reconstruction in anisotropic media, Ultrasonics 41, 125-131 (2003).

[8] S. Rodriguez, M. Castaings, M. Deschamps, and E. Ducasse, Topological imaging of defects in anisotropic plates, in V. Le Cam, L. Mevel, and F. Schoefs, editors, EWSHM - rth European Workshop on Structural Health Monitoring, pages 1155-1162 (2014).

[9] D. Clorennec, C. Prada, and D. Royer, Local and noncontact measurements of bulk acoustic wave velocities in thin isotropic plates and shells using zero group velocity Lamb modes, J. Appl. Phys. 101, 034908 (2007).

[10] D. Clorennec, C. Prada, and D. Royer, Laser ultrasonic inspection of plates using zero-group velocity Lamb modes, IEEE Trans. Ultrason. Ferroelectr. Freq. Control. 57, 1125-1132 (2010).

[11] J. F. Ready, in J. F. Ready, editor, Effects of High-Power Laser Radiation, chapter 3, pages 67-125, Academic Press (1971).

[12] O. Balogun, T. W. Murray, and C. Prada, Simulation and measurement of the optical excitation of the $S_{1}$ zero group velocity Lamb wave resonance in plates, J. Appl. Phys. 102, 064914 (2007).

[13] J. A. Rogers and K. A. Nelson, Study of lamb acoustic waveguide modes in unsupported polyimide thin films using real-time impulsive stimulated thermal scattering, J. Appl. Phys. 75, 1534-1556 (1994). 
[14] J. A. Rogers, A. A. Maznev, M. J. Banet, and K. A. Nelson, Optical generation and characterization of acoustic waves in thin films: fundamentals and applications, Annu. Rev. Mater. Sci. 30, 117-157 (2000).

[15] A. A. Maznev and A. G. Every, Surface acoustic waves with negative group velocity in a thin film structure on silicon, Appl. Phys. Lett. 95, 011903 (2009).

[16] A. Bennis, A. M. Lomonosov, Z. H. Shen, and P. Hess, Laser-based measurement of elastic and mechanical properties of layered polycrystalline silicon structures with projection masks, Appl. Phys. Lett. 88, 101915 (2006).

[17] C. Grünsteidl, I. A. Veres, J. Roither, P. Burgholzer, T. W. Murray, and T. Berer, Spatial and temporal frequency domain laser-ultrasound applied in the direct measurement of dispersion relations of surface acoustic waves, Appl. Phys. Lett. 102, 011103 (2013).

[18] F. Reverdy and B. Audoin, Elastic constants determination of anisotropic materials from phase velocities of acoustic waves generated and detected by lasers, J. Acoust. Soc. Am. 109, 1965-1972 (2001).

[19] B. Hosten and B. Castagnede, Optimisation du calcul des constantes élastiques à partir des mesures de vitesses d'une onde ultrasonore, $C . R$. Acad. Sc. Paris, 296, 297-300 (1983).

[20] A. L. Shuvalov and O. Poncelet, On the backward lamb waves near thickness resonances in anisotropic plates, Int. J. Sol. Struct. 45, 34303448 (2008). 
[21] T. Hussain and F. Ahmad, Lamb modes with multiple zero-group velocity points in an orthotropic plate, J. Acoust. Soc. Am. 132, 641-645 (2012).

[22] S. Raetz, J. Laurent, T. Dehoux, D. Royer, B. Audoin, and C. Prada, Effect of refracted light distribution on the photoelastic generation of zero-group velocity Lamb modes in optically low-absorbing plates, $J$. Acoust. Soc. Am. 138, 3522-3530 (2015).

[23] R. K. Ing and J. Monchalin, Broadband optical detection of ultrasound by two-wave mixing in a photorefractive crystal, Appl. Phys. Lett. 59, 3233-3235 (1991).

[24] B. Campagne, H. Voillaume, L. Gouzerh, and F. Bentouhami, Laser ultrasonic developments for NDT of aeronautic composite parts, in 13th International Symposium on Nondestructive Characterization of Materials (NDCM-XIII) 2013, Le Mans, France, volume 19, NDT.net, The e-Journal of Nondestructive Testing \& Ultrasonics (2014).

[25] P. Cawley and C. Theodorakopoulos, The membrane resonance method of non-destructive testing, J. Sound Vibrat. 130, 299-311 (1989).

[26] I. Solodov, J. Bai, S. Bekgulyan, and G. Busse, A local defect resonance to enhance acoustic wave-defect interaction in ultrasonic nondestructive evaluation, Appl. Phys. Lett. 99, 211911 (2011). 


\section{List of Figures}

1 (color online) Dispersion curves of the symmetric (solid line) and antisymmetric (dashed line) Lamb modes calculated in a 3.2-mm-thick composite plate. The first ZGV mode is shown by the vertical arrow and has a wavelength of $\lambda_{Z G V}^{c o m p} \approx 15.3 \mathrm{~mm} . \quad 6$

2 (color online) Illustration of the ZGV Lamb mode generation using a beam-shaping mask. The generation laser beam is shaped with periodic slits whose spacing matches the ZGV wavelength. ................... . . 7

3 (color online) Schematics of the experimental transmission setup. 9

4 (color online) The thermoelastic source is a line $4.1 \times 20 \mathrm{~mm}^{2}$ :

(a) time domain and (b) frequency domain B-scans as a function of the TWM head position. (c) First ZGV peak amplitude vs. TWM head position: experimental (solid) and theoretical (dashed) curves. . . . . . . . . . . . . . . . . 11

5 (color online) Normalized first ZGV peak amplitude vs. TWM head position when the thermoelastic source is a single line (solid), multiple lines spaced by $\lambda_{Z G V}^{A l}$ (dashed) and multiple lines spaced by $\lambda_{Z G V}^{A l} / 2$ (dash-dotted). . . . . . . . . . . . 12

6 (color online) Results obtained in the composite plate with a thermoelastic source made of three lines: (a) time domain and (b) frequency domain B-scans vs. TWM head position. (c) Normalized amplitude of the first ZGV peak vs. TWM head position (thick line) compared with the result obtained with a single line source (thin line). . . . . . . . . . . . . 15 
7 (color online) Composite sample and representation of the scan lines (solid). Dashed white line circle: maximum size of the flaw observed optically. . . . . . . . . . . . . . . . . . 16

8 (color online) Time domain B-scans of the composite plate when the scan line is: line 1 across the impact location (a), line 2 near the impact location (b), and line 3 far from the impact location (c). The related frequency domain B-scans obtained with a Hann time window (respectively d, e and f). Dashed white lines: maximum dimension of the flaw observed optically. . . . . . . . . . . . . . . . . 17

9 (color online) C-scan inspection of the composite plate obtained with the LUCIE system: ratio of the second ultrasonic echo amplitude to the first ultrasonic echo amplitude . . . . . 20 